\title{
PHASE TRANSITION AND HYPERSCALING VIOLATION FOR SCALAR BLACK BRANES
}

\author{
Mariano Cadoni \\ Dipartimento di Fisica, Università di Cagliari and INFN, \\ Sezione di Cagliari - Cittadella Universitaria, 09042 Monserrato, Italy. \\ Salvatore Mignemi \\ Dipartimento di Matematica, Università di Cagliari and INFN, \\ Sezione di Cagliari - viale Merello 92, 09123 Cagliari, Italy.
}

(Dated: November 16, 2018)

\begin{abstract}
We investigate the thermodynamical behavior and the scaling symmetries of the scalar dressed black brane (BB) solutions of a recently proposed, exactly integrable Einstein-scalar gravity model [1], which also arises as compactification of $(p-1)$-branes with a smeared charge. The extremal, zero temperature, solution is a scalar soliton interpolating between a conformal invariant AdS vacuum in the near-horizon region and a scale covariant metric (generating hyperscaling violation on the boundary field theory) asymptotically. We show explicitly that for the boundary field theory this implies the emergence of an UV length scale (related to the size of the brane), which decouples in the IR, where conformal invariance is restored. We also show that at high temperatures the system undergoes a phase transition. Whereas at small temperature the Schwarzschild-AdS BB is stable, above a critical temperature the scale covariant, scalar-dressed BB solution, becomes energetically preferred. We calculate the critical exponent $z$ and the hyperscaling violation parameter $\theta$ of the scalar-dressed phase. In particular we show that $\theta$ is always negative. We also show that the above features are not a peculiarity of the exact integrable model of Ref. [1], but are a quite generic feature of Einstein-scalar and Einstein-Maxwell-scalar gravity models for which the squared-mass of the scalar field $\phi$ is positive and the potential vanishes exponentially as $\phi \rightarrow-\infty$.
\end{abstract}

\section{INTRODUCTION}

In recent years holographic methods have been widely used to investigate features of strongly interacting quantum field theories (QFT), in particular for what concerns possible applications to condensed matter systems [2-11].

These holographic methods have been developed following rather closely the anti-de Sitter/conformal field theory (AdS/CFT) correspondence paradigm. This means that the focus has been mainly on bulk AdS gravity in $d+2$ dimensions and its $(d+1)$-dimensional conformal field theory duals. In fact, it has turned out that simply putting in the AdS background a black hole (black brane), a non trivial scalar field configurations and eventually finite electromagnetic charge density, a very rich phenomenology in the dual QFT could be obtained. This includes spontaneous breaking of the $U(1)$ symmetry, phase transitions triggered by scalar condensates and non-trivial transport properties of the dual field theory [2 15].

An interesting and welcome byproduct of these investigations has been the realization of the importance played by non-AdS gravitational backgrounds and the related dual nonconformal QFTs. When the self-interaction potential and the Maxwell tensor/scalar field coupling function behave exponentially, a quite generic feature of the bulk Einstein-Maxwell-scalar gravity theory is the emergence in the extremal, near-horizon (infrared) region of solutions breaking the full conformal symmetry of the (ultraviolet) AdS vacuum, but still preserving some symmetry of the AdS background $^{1}$ [8, 9, 11, 13, 16 21].

The existence of these non-AdS solution is not just a peculiarity of theories with non minimal

1 Throughout this paper we use infrared (IR) and ultraviolet (UV) to denote, respectively, the infrared and ultraviolet region of the dual boundary field theory. In terms of the bulk gravity theory they correspond respectively to the near-horizon and asymptotic region. 
(exponential) couplings between the scalar and the Maxwell field 7 $-9,11,22$ 25]. They also arise as solutions of string theory and supergravity constructions [20,26 28]. From a purely gravitational point of view their existence is a generic consequence of the presence of a non-trivial scalar with a self interaction potential behaving exponentially [29].

According to the kind of symmetry that is preserved in the IR, these non-AdS metrics can be classified in two classes. To the first class, called Lifshitz, belong metrics for which a scale isometry, under which timelike and spacelike coordinates transform with a different exponent, is preserved but the conformal and Poincaré isometry of the $d+1$-dimensional spacetime is broken $[10,11,17,30$ 33]. They could be relevant for the holographic description of quantum phase transitions. In analogy with critical systems in condensed matter physics, such metrics are characterized by a dynamical critical exponent $z$ describing the anisotropy of scaling of the space and time coordinates. The second class of these non-AdS metrics is characterized by breaking of the scale isometry, but the $(d+1)$-dimensional Poincaré invariance is preserved. In the literature they are often referred to as domain wall (DW) solutions [1, 26, 29, 34, 35], in analogy with the DW solutions of supergravity (SUGRA) theories.

Very recently, it has been realized that these two classes of metrics are particular cases of a more general class of metrics that are not scale invariant (but only scale covariant) [10, 13, 29] and lead to hyperscaling violation in the dual field theory [19, 20, 36]. They are characterized by two parameters, the critical exponent $z$ and the hyperscaling violation parameter $\theta$, which gives the transformation weight of the infinitesimal length $d s$ under scale transformations and the corresponding scaling behavior of the free energy as function of the temperature [20, 36]. Scale covariant metrics are a very promising framework for the holographic description of hyperscaling violation in condensed matter critical systems (e.g Ising models) 37].

The crucial holographic feature of the class of models allowing for scale-covariant metrics, is the emergence of a length scale in the IR [20]. If the theory has an UV fixed point, this length scale decouples in the UV because of the conformal invariance of the AdS background. The emergence of a length scale in the IR has several interesting physical consequences, which have been used for the description of Fermi surfaces and for the related area-law violation of entanglement entropy 20, 36, 38, 39].

Until now the standard setup used for obtaining, dynamically, scale -covariant metrics, is given by Einstein-scalar gravity, possibly coupled - minimally or non-minimally - with a $U(1)$ field. The selfinteraction potential $V(\phi)$ for the scalar field $\phi$ is assumed to have a negative local maximum at $\phi=$ 0 , with a corresponding scalar tachyonic excitation whose mass is slightly above the BreitlohnerFreedman (BF) bound. The $\phi=0$ solution of the gravity theory corresponds to the AdS vacuum, whereas under suitable conditions - typically an exponentially behavior of the potential and/or scalar/Maxwell tensor coupling functions - the theory admits black brane solutions with scalar hair that in the near-extremal regime approach the scale covariant metric.

The requirement that the $\phi=0$ solution be a maximum of $V(\phi)$ is necessary for the existence of non trivial black brane solutions with AdS asymptotics. In fact, usual no-hair theorems based on the positive energy theorem forbid the existence of black brane (BB) solutions with AdS asymptotics when the squared-mass of the scalar is positive [40 42]. However, in a recent paper it has been shown that these no-hair theorems can be circumvented by giving up the condition that the BB solution has AdS asymptotics [1, 29]. In Ref. [1] we have derived exact hairy, asymptotically non-AdS, black brane solutions of a Einstein-scalar gravity model with positive squared mass for the scalar field. We have shown that the extremal limit of these BB solutions is a scalar soliton which interpolates between an AdS vacuum in the near-horizon region and a scale covariant, DW, solution in the asymptotic region. Moreover, these BB solutions arise as Kaluza-Klein compactification of black 2-branes with a smeared charge supported by a 4 -form field strength [10].

The results of Refs. [1, 10] open up the possibility of realizing an alternative scenario in which the usual roles played by the IR and UV regions are reversed. In the dual QFT, the IR physics is determined by an infrared fixed point, i.e. by the conformal symmetry of the AdS vacuum, whereas the UV behavior is characterized by hyperscaling violation and by an emergent UV length scale. In this paper we study in detail this alternative framework. Building on the results of Ref. [1], we first show that the scalar soliton obtained as the zero-temperature extremal limit 
of the BB solutions of Ref. [1] gives a nice realization of this scenario (Section [II). Using the Euclidean action formalism we derive a consistent formulation for the thermodynamics of our BB solutions (Section III). This allows us to show that in these Einstein-scalar gravity models the Schwarzschild-anti de Sitter (SAdS) solution is unstable above a critical temperature $T_{c}$. At small temperatures the SAdS solution with constant vanishing scalar field is energetically preferred with respect to a scalar-dressed, BB solution with DW asymptotics. This is not the case for $T>T_{c}$, and the thermodynamical system undergoes a first-order phase transition SAdS $\rightarrow$ scalar BB solution (Section IV). The thermodynamical behavior of the solutions, the emergence of a length scale in the UV and its decoupling in the IR have a natural explanation in terms of the symmetries of the solutions. In particular, we show that the asymptotic solution is a scale-covariant metric characterized by critical exponent $z=1$ and negative hyperscaling-violating parameter $\theta$ (Section V). Although most of the calculations have been performed for the exact BB solution of the Einstein-scalar gravity model introduced in Ref. 1], we show that the most important features (SAdS $\rightarrow$ scalar BB phase transition, hyperscaling violation) are quite generic features of a broad class of Einstein-scalar and Einstein-Maxwell-scalar gravity models. Sufficient conditions for their presence are the existence of a negative minimum of the potential $V(\phi)$ and an exponential behavior, $V(\phi) \sim e^{\alpha \phi}, \alpha>0$, for $\phi \rightarrow-\infty$ (Section VII).

\section{BLACK BRANE SOLUTIONS}

We consider static, radially symmetric, planar solutions of Einstein gravity minimally coupled to a scalar field with self-interaction potential $V(\phi)$. The action is

$$
I=\frac{1}{16 \pi G} \int d^{4} x\left[R-2(\partial \phi)^{2}-V(\phi)\right] .
$$

where $\phi$ is a scalar field. Following widespread conventions we set $G=1 /(16 \pi)$.

We will consider models with a potential $V(\phi)$ satisfying the following conditions: 1) $V(\phi)$ has a local minimum for $\phi=0$ with $V(0)<0 ; 2)$ The potential approaches zero exponentially as $\phi \rightarrow-\infty$. We will therefore assume the following behavior,

$$
V(\phi) \sim e^{2 h \phi}, h>0, \text { for } \phi \rightarrow-\infty ; \quad V(0)=-\frac{6}{L^{2}}, V^{\prime}(0)=0, V^{\prime \prime}(0)>0,
$$

where $L$ is the AdS length. The previous conditions ensure the existence of an $\mathrm{AdS}_{4}$ vacuum and of a Schwarzschild-AdS (SAdS) black brane solution with $\phi=0$. On the other hand standard positive energy theorems forbid the existence of black brane solutions with scalar hair and AdS asymptotics [40 42].

We first focus on a particular model, which is exactly integrable. In sectionVII we will extend our considerations to general models satisfying (2.2). The particular model we consider is characterized by the potential

$$
V(\phi)=-\frac{6}{\gamma L^{2}}\left(e^{2 \sqrt{3} \beta \phi}-\beta^{2} e^{\frac{2 \sqrt{3}}{\beta} \phi}\right), \quad \gamma=1-\beta^{2},
$$

where $\beta$ is a real parameter. The limit $\beta \rightarrow 1$ gives a Liouville (purely exponential) potential, whereas for $\beta=0$ the potential reduces to a cosmological constant. The action is invariant under the duality transformation $\beta \rightarrow 1 / \beta$.

The gravity-scalar model defined by the potential (2.3) has several interesting features. It is a fake SUGRA model, i.e. the potential can be derived from a superpotential $P(\phi)=$ $(1 / \gamma L)\left(e^{\sqrt{3} \beta \phi}-\beta^{2} e^{\frac{\sqrt{3}}{\beta} \phi}\right)$, see Ref. [1]. The model is exactly integrable, since the field equations for static, radially symmetric, planar solutions can be reduced to that of a Toda molecule. The model allows to circumvent standard no-hair theorems - it admits black brane solutions with non-AdS asymptotics. Last but not least, the model (2.3) arises as Kaluza-Klein compactification 
on a $q$-dimensional compact space $\mathbf{K}^{q}$ of a black $(p-1)$-brane with a smeared charge, which is solution of the action [10],

$$
S=\int d^{p+q+1} x \sqrt{-g}\left(R-\frac{1}{2(n+2) !} G_{(n+2)}\right)
$$

where $G_{(n+2)}$ is the field-strength form. The action (2.1) with the potential (2.3) is obtained by Kalunza-Klein compactification of $\mathbf{K}^{q}$ considering $p=3$, i.e a 2-brane, with $n=p-1=2$, so that $G_{(n+2)}$ can be dualized to a scalar [10].

The potential (2.2) has a minimum at $\phi=0$ with $V(0)=-6 / L^{2}$, corresponding to an $\mathrm{AdS}_{4}$ vacuum and related local scalar excitation of positive squared-mass $m^{2}=18 / L^{2}$. For $\phi \rightarrow-\infty$ the potential approaches zero, while it diverges for $\phi \rightarrow \infty$. The plot of $V(\phi)$ for a selected value of the parameter, $\beta=1 / 2$ and $L=1$, is shown in Fig. 1.

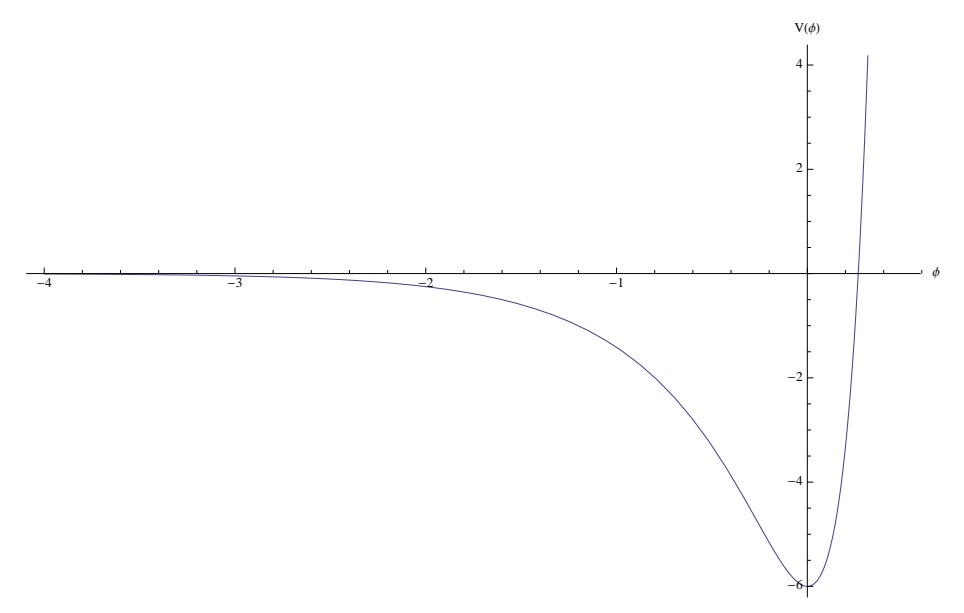

FIG. 1: Plot of the potential $V(\phi)$ for a selected value of the parameter $\beta=1 / 2$ and $L=1$.

It has been shown in [1] that the present model admits two classes of static black brane solutions: the SAdS black brane with constant vanishing scalar field and a scalar black brane endowed with a non trivial, $r$-dependent, scalar field profile.

The SAdS solution always exists for every value of $\beta$. The scalar field is $\phi=0$ (minimum of the potential $V$ ) and the metric part of the solution reads

$$
d s^{2}=-f(r) d t^{2}+f^{-1}(r) d r^{2}+r^{2} d x_{i} d x_{i}, \quad f(r)=\frac{r^{2}}{L^{2}}-\frac{2 M}{r} .
$$

where $M$ is the black brane mass.

We now investigate the solution with non-trivial scalar field, starting from the values $\beta^{2}<1$. The case $\beta^{2}>1$ will be discussed in Sect. VI.

For $\beta^{2}<1$, the black brane solution is given by ${ }^{2}$

$$
d s^{2}=\Delta(r)^{\frac{2 \beta^{2}}{3 \gamma}}\left[\left(\frac{r}{r_{0}}\right)^{\frac{2}{1+3 \beta^{2}}}\left(-\Gamma(r) d t^{2}+d x_{i} d x_{i}\right)+E \frac{\Delta(r)^{\frac{4 \beta^{2}}{3 \gamma}}}{\Gamma(r)}\left(\frac{r}{r_{0}}\right)^{-\frac{2}{1+3 \beta^{2}}} d r^{2}\right]
$$

\footnotetext{
2 The solution in this form, apart from some trivial change of notation, has been derived in Ref. [1]. The same solution, albeit in a different form, is a particular case $(p=3, n=2)$ of the compactified black $(p-1)$-brane solutions with a smeared charge derived in Ref. [10].
} 


$$
e^{2 \phi}=\left[\frac{A}{\Delta(r)}\left(\frac{r}{r_{0}}\right)^{-\frac{3 \gamma}{1+3 \beta^{2}}}\right]^{\frac{2 \beta}{\sqrt{3} \gamma}},
$$

where $\Gamma(r)=1-\mu_{1}\left(\frac{r_{0}}{r}\right)^{\delta}, \Delta(r)=1+\mu_{2}\left(\frac{r_{0}}{r}\right)^{\delta}, \delta=3 \gamma /\left(1+3 \beta^{2}\right), A=\sqrt{\mu_{2}\left(\mu_{1}+\mu_{2}\right)}, E=$ $\left(\frac{\gamma L}{\left(1+3 \beta^{2}\right) r_{0}}\right)^{2} A^{-2 \beta^{2} / \gamma}, \mu_{1,2}$ are dimensionless free parameters and $r_{0}$ is a length scale that must be introduced in order to get the correct physical dimensions. Physically, $r_{0}$ is related to the size of the brane. In fact, it drops out completely from the solution (2.6) just by rescaling appropriately the coordinate $x_{i}$ (and the time $t$ ) and by redefining the parameters $\mu_{1,2}$. In principle $r_{0}$ can take any value, but for a holographic interpretation to hold, we require $r_{0} \gg L$. The parameters $\mu_{1} \geq 0$ and $\mu_{2} \geq 0$ are assumed to be small numbers, even if in principle $\mu_{1}$ can have a greater scale than $\mu_{2}$. Note that the metric functions $g_{t t}$ and $g_{i i}$ can be arbitrarily rescaled, since no natural normalization exists for the coordinates $t$ and $x_{i}$.

The asymptotic behavior of the solution (2.6) for $r \rightarrow \infty$ is that of a domain wall: $d s^{2}=$ $\left(r / r_{0}\right)^{\eta}\left(-d t^{2}+d x_{i} d x_{i}\right)+\left(r / r_{0}\right)^{-\eta} d r^{2}$, where $\eta=2 /\left(1+3 \beta^{2}\right)$, and the scalar field behaves logarithmically, $\phi=-\left[(\sqrt{3} \beta) /\left(1+3 \beta^{2}\right)\right] \log \left(r / r_{0}\right)$. One may also define a scalar charge, which is proportional to $\mu_{2}$.

For $\mu_{1}>0$, the metric (2.6) exhibits a singularity at $r=0$, shielded by a horizon at $r / r_{0}=\mu_{1}^{1 / \delta}$, and therefore represents a regular black brane. Owing to the fact that the scalar $\phi$ depends on $\mu_{1}$, the existence of this black brane solution is perfectly consistent with the no-hair theorem of Ref. [29]. Notice that for $\mu_{2}>0$, although the scalar field remains finite at $r=0$, the scalar curvature $R$ of spacetime diverges as $R \sim r^{-3\left(1+\beta^{2}\right) /\left(1+3 \beta^{2}\right)}$. For $\mu_{2}=0$ the metric (2.6) describes the black brane solution with a $\phi \sim \ln r$ short distance singularity discussed in Ref. 29].

A detailed discussion of the causal structure of the spacetimes (2.6) is beyond the scope of this paper. We just note that the causal structure of two-dimensional models with metric behaving as $d s^{2}=-r^{a} d t^{2}+r^{-a} d r^{2}$ with $0<a \leq 2$, has been already discussed in Ref. [43]. From those results it follows immediately that the $r=\infty$ asymptotic region is spacelike for $\beta^{2} \geq 1 / 3$, whereas it becomes timelike for $\beta^{2}<1 / 3$. The timelike boundary at $r=\infty$, for $\beta^{2}<1 / 3$, makes the spacetime conformally equivalent to $\mathrm{AdS}_{4}$, and is therefore a crucial ingredient for a holographic interpretation of these solutions [26, 34, 35]. Notice that in the coordinates used in Eq. (2.6), $r \rightarrow \infty$ describes the UV regime of the dual QFT, whereas $r \rightarrow 0$ corresponds to the IR regime.

The limiting case $\beta^{2}=1 / 3$ of the solution (2.6) is particularly simple:

$$
\begin{aligned}
d s^{2} & =\left(1+\mu_{2} \frac{r_{0}}{r}\right)\left[-\left(r-\mu_{1} r_{0}\right) d t^{2}+\frac{E\left(1+\mu_{2} \frac{r_{0}}{r}\right)^{2}}{\left(r-\mu_{1} r_{0}\right)} d r^{2}+r d x_{i} d x_{i}\right], \\
e^{2 \phi} & =\frac{A}{r+\mu_{2} r_{0}} .
\end{aligned}
$$

Asymptotically, the two-dimensional $x_{i}=$ const. sections of the metric (2.7) describe Rindler spacetime.

For $\mu_{2}>0$, the extremal limit $\mu_{1}=0$ of the solution (2.6) represents a regular scalar soliton [1], i.e. a soliton endowed with a non trivial scalar profile. In fact, not only the scalar field vanishes at $r=0$, but also the scalar curvature of the spacetime remains finite both at $r=0$ and $r=\infty$. The scalar soliton does not exist for $\mu_{2}=0$. In this case the extremal limit $\mu_{1}=0$ is a spacetime with a singularity at $r=0$ and a scalar field behaving as $\log r$.

In order to clarify the role played by the two scales $L$ and $r_{0}$, let us now write explicitly the form of the solitonic solution. Setting $\mu_{1}=0$, the metric (2.6) becomes

$$
\begin{aligned}
d s^{2} & =\Delta(r)^{\frac{2 \beta^{2}}{3 \gamma}}\left[\left(\frac{r}{r_{0}}\right)^{\frac{2}{1+3 \beta^{2}}}\left(-d t^{2}+d x_{i} d x_{i}\right)+k^{2}\left(\frac{L}{r_{0}}\right)^{2} \Delta(r)^{\frac{4 \beta^{2}}{3 \gamma}}\left(\frac{r}{r_{0}}\right)^{-\frac{2}{1+3 \beta^{2}}} d r^{2}\right], \\
e^{2 \phi} & =\left[\frac{\mu_{2}}{\Delta(r)}\left(\frac{r}{r_{0}}\right)^{-\frac{3 \gamma}{1+3 \beta^{2}}}\right]^{\frac{2 \beta}{\sqrt{3} \gamma}}
\end{aligned}
$$


where $k=\gamma /\left(1+3 \beta^{2}\right) \mu_{2}^{-\beta^{2} / \gamma}$.

In the IR limit, $r \rightarrow 0$, the scalar field approaches to zero, the length scale $r_{0}$ decouples and the metric (2.8) becomes, after suitable rescaling of $t$ and $x_{i}$, the metric of $\mathrm{AdS}_{4}$, with AdS length $L$ :

$$
d s^{2}=\frac{r^{2}}{L^{2}}\left(-d t^{2}+d x_{i} d x_{i}\right)+\frac{L^{2}}{r^{2}} d r^{2} .
$$

Note that, because of scale invariance, $g_{r r}$ is independent from the scale of $r$. In this regime, that holds for $r \lesssim L$, conformal invariance is restored.

Conversely, in the UV limit $r \rightarrow \infty$, it is the AdS length $L$ that decouples, and the metric (2.6) can be written entirely in terms of $r_{0}$ only. In fact taking in Eq. (2.8) the limit $r \rightarrow \infty$ one gets, after suitable rescaling of $r, t, x_{i}$, the scale-covariant domain wall metric

$$
d s^{2}=\left(\frac{r}{r_{0}}\right)^{\frac{2}{1+3 \beta^{2}}}\left[-d t^{2}+d x_{i} d x_{i}\right]+\left(\frac{r}{r_{0}}\right)^{-\frac{2}{1+3 \beta^{2}}} d r^{2},
$$

whereas the scalar field behaves logarithmically $\phi=-\left[(\sqrt{3} \beta) /\left(1+3 \beta^{2}\right)\right] \log \left(r / r_{0}\right)$. This regime is attained for $r \gg r_{0}$. In this case, the form of $g_{r r}$ depends on the rescaling or $r$. The metric (2.10) is not invariant under scale transformations but transforms with a definite weight.

The extremal soliton has therefore the form of a brane that interpolates between a scale covariant, domain wall solution in the UV and the AdS spacetime in the IR. The metric behaves as $d s^{2}=$ $-\left(r / l_{a}\right)^{\eta}\left[-d t^{2}+d x_{i} d x_{i}\right]+\left(r / l_{a}\right)^{-\eta} d r^{2}$ in the limit of small or large $r$, with different power $\eta$ and length-scale $l_{a}$ in the IR and UV. This behavior has a natural interpretation in terms of the symmetries of the solutions (2.9) and (2.10) as we will explain in detail in Sect. V

It is also important to notice that the $r \rightarrow \infty$ UV region of the soliton (2.6), corresponds to $\phi \rightarrow-\infty$, the region where the potential $V(\phi)$ approaches zero. Conversely, the $r=0$ IR region corresponds to $\phi=0$, i.e. to the minimum of the potential $V(\phi)$.

It is instructive to think of the above-described situation as the opposite (IR exchanged with UV) of that holding in Einstein-gravity models with holographic applications to condensed matter systems. Typically, in the latter case one considers potentials $V(\phi)$ with a negative maximum and with the negative squared-mass $m^{2}$ of the corresponding tachyonic excitation slightly above the BF bound. In this case the dual field theory has an UV fixed point, corresponding to the AdS vacuum and the IR corresponds to strong self-interaction of the scalar $V(\phi)=-\infty$. The length-scale $r_{0}$ is an IR scale, which decouples in the UV, where conformal invariance is restored.

Let us now come back to the general solution (2.6) with $\mu_{1} \neq 0$. Because (2.6) is a global solution interpolating between the IR and the UV regime and because the two regimes are characterized by two different length scales $r_{0}$ and $L$, it is convenient to define dimensionless coordinates $t, r$. Moreover this will allow us to write the solution in a simpler form. Rescaling the coordinates as follows,

$$
\frac{r}{r_{0}} \rightarrow r D^{\left(1+3 \beta^{2}\right) / 3 \gamma}, \quad t \rightarrow\left(\frac{\gamma L}{1+3 \beta^{2}}\right) t, \quad x_{i} \rightarrow x_{i} D^{-1 / 3 \gamma}
$$

the solution (2.6) takes the form,

$$
\begin{aligned}
d s^{2} & =\left(\frac{\gamma L}{1+3 \beta^{2}}\right)^{2}\left[-\Delta^{\frac{2 \beta^{2}}{3 \gamma}}(r)\left(1-\frac{\nu_{1}}{r^{\delta}}\right) r^{\frac{2}{1+3 \beta^{2}}} d t^{2}+\frac{\Delta^{\frac{2 \beta^{2}}{\gamma}}(r) d r^{2}}{\left(1-\frac{\nu_{1}}{r^{\delta}}\right) r^{\frac{2}{1+3 \beta^{2}}}}\right]+\Delta^{\frac{2 \beta^{2}}{3 \gamma}}(r) r^{\frac{2}{1+3 \beta^{2}}} d x_{i} d x_{i}, \\
e^{2 \phi} & =\Delta^{-\frac{2 \beta}{\sqrt{3} \gamma}}(r) r^{-\frac{2 \sqrt{3} \beta}{1+3 \beta^{2}}},
\end{aligned}
$$

where now $\Delta(r)=1+\frac{\nu_{2}}{r^{\circ}}$ and $\nu_{1,2}=\frac{\mu_{1,2}}{A}=\frac{\mu_{1,2}}{\sqrt{\mu_{2}\left(\mu_{2}+\mu_{1}\right)}}$.

Unfortunately, the dimensionless parameters $\nu_{1}$ and $\nu_{2}$ so defined are not independent, but are constrained by the algebraic relation

$$
\nu_{2}\left(\nu_{2}+\nu_{1}\right)=1
$$


which can solved for $\nu_{1}$ :

$$
\nu_{1}=\frac{1}{\nu_{2}}-\nu_{2}
$$

Thus, in the new coordinates the solution has only one independent parameter, $\nu_{2}$. This means that one cannot vary independently the black brane temperature (or mass) and the scalar charge. For instance, the $T=0$ scalar soliton has necessarily $\nu_{2}=1$. The range of variation of $\nu_{2}$ is $0 \leq \nu_{2} \leq 1$, corresponding to $\infty \geq \nu_{1} \geq 0$.

Notice that the solution (2.12) and the constraint (2.13) can be directly derived from the general solution (2.6) by imposing that the scalar field does not depend explicitly on $\nu_{1}$. This requires $A=1$, which is equivalent to Eq. (2.13). With this assumption we get, after a rescaling of the time coordinate, solution (2.12) from solution (2.6).

The two forms of the solution are completely consistent with the no-hair theorem of Ref. 29]. In the form (2.6) the solution is parametrized by two independent parameters $\mu_{1,2}$ but the scalar field depends on both of them, i.e. it depends on both the black brane temperature and the scalar charge $\mu_{2}$. When we try to make the scalar field independent from the temperature we are forced to impose the constraint (2.13) and the solution has only one independent parameter. The scalar field depends now implicitly on the temperature. In the form (2.12) our solution is very similar to that of Martinez et al. 44. Indeed, both solutions fully confirm the no-hair theorem or Ref. 29].

As we shall see, the thermodynamics of the black brane can be consistently defined only for the metric (2.12). It is an open question if to the two-parameter solution (2.6) can be given a physical interpretation. Its existence follows from the fact that the angular part of the metric has no natural normalization in planar coordinates.

\section{THERMODYNAMICS}

To set up the thermodynamics of our black brane solutions we use the euclidean action formalism of Martinez et al. [44] (Our conventions in the action (2.1) correspond to setting $G=1 / 16 \pi$ and to multiplying by 4 the kinetic term for the scalar field in the paper of Martinez et al.).

The thermodynamical behavior of the solutions (2.6) is problematic due to the occurrence of divergences in the boundary action, that determines the mass of the solution. This divergences are difficult to remove because the scalar depends explicitly on the parameter $\mu_{1}$. On the other hand, as we will show later in this section, the formulation of the thermodynamics of the solution (2.12) with the parameters $\nu_{1,2}$ constrained by Eq. (2.13) is free from this difficulty. It is not completely clear to us whether a consistent thermodynamical interpretation of the two-parameter solution (2.6) exists. We will not address this issue here but we will simply formulate the thermodynamics of the solution written in the form (2.12), (2.13). In the calculation we will first consider $\nu_{1,2}$ as independent parameters, and then use the constraint (2.14) at the end of the calculations.

Standard calculations give for the temperature $T$ and entropy $S$ of our black brane solution (2.12)

$$
T=\frac{1}{4 \pi} \frac{3 \gamma}{1+3 \beta^{2}}\left(\nu_{1}+\nu_{2}\right)^{-\frac{2 \beta^{2}}{3 \gamma}} \nu_{1}^{1 / 3}, \quad S=\frac{\Omega R^{2}}{4 G^{2}}=4 \pi \Omega\left(\nu_{1}+\nu_{2}\right)^{\frac{2 \beta^{2}}{3 \gamma}} \nu_{1}^{2 / 3}
$$

where $\Omega$ is the volume of the transverse 2-dimensional space. Since we are working with dimensionless coordinates (see the metric (2.12) ) the black brane temperature and energy are also dimensionless. The physical dimensions can however easily be restored inverting (2.11). Note that the scale of the temperature depends on the normalization of the time coordinates $t$, which is arbitrary.

In the Euclidean action formalism, the thermodynamical potentials are given as boundary terms of the action at infinity and on the horizon $r=r_{h}$. Using the parametrization of the metric,

$$
d s^{2}=N^{2} \Lambda d t^{2}+\frac{d r^{2}}{\Lambda}+R^{2} d x_{i} d x^{i}
$$


the gravitational and scalar part of the variation of the boundary terms are given respectively by [44]

$$
\begin{aligned}
\delta I_{G} & =\left.\frac{2 \Omega}{T}\left[N\left(R R^{\prime} \delta \Lambda-\Lambda^{\prime} R \delta R\right)+2 \Lambda R\left(N \delta R^{\prime}-N^{\prime} \delta R\right)\right]\right|_{r_{h}} ^{\infty}, \\
\delta I_{\phi} & =\left.\frac{4 \Omega}{T} N R^{2} \Lambda \phi^{\prime} \delta \phi\right|_{r_{h}} ^{\infty} .
\end{aligned}
$$

The contributions of the two boundaries at $r=\infty$ and $r=r_{h}$ are given by

$$
\begin{aligned}
\delta I_{G}^{\infty} & =-\frac{2 \Omega}{T\left(1+3 \beta^{2}\right)}\left[\delta \nu_{1}+\frac{2 \beta^{2}}{\gamma}\left(2-\beta^{2}\right) \delta \nu_{2}\right], \\
\delta I_{\phi}^{\infty} & =\frac{4 \Omega \beta^{2}}{\gamma T\left(1+3 \beta^{2}\right)} \delta \nu_{2}, \\
\left.\delta I_{G}\right|_{r_{h}} & =\frac{2 \Omega}{T\left(1+3 \beta^{2}\right)} \frac{1}{\left(\nu_{1}+\nu_{2}\right)}\left[\left(\nu_{1}+\gamma \nu_{2}\right) \delta \nu_{1}+\beta^{2} \nu_{1} \delta \nu_{2}\right], \\
\left.\delta I_{\phi}\right|_{r_{h}} & =0 .
\end{aligned}
$$

One can easily show that $\left.\delta I_{G}\right|_{r_{h}}=4 \pi \Omega \delta R^{2}\left(r_{h}\right)=\delta S$. This gives the entropy

$$
S=\left.I_{G}\right|_{r_{h}}
$$

On the other hand, because $\left.\delta I_{\phi}\right|_{r_{h}}=0$, there is no thermodynamical potential associated to the scalar field. From the definition of the the free energy $F=M-T S$ and from $F=-I T$, it follows $M=T S-T I=-T\left(\delta I_{G}^{\infty}+\delta I_{\phi}^{\infty}\right)$. Using Eqs. (3.5) one finds

$$
M=\frac{2 \Omega}{1+3 \beta^{2}}\left(\nu_{1}+2 \beta^{2} \nu_{2}\right) .
$$

To check the correctness of our calculations, let us consider two particular cases, $\beta=0$ and $\nu_{2}=0$. For $\beta=0$ our solution becomes the Schwarzschild-AdS black brane and it is easily seen that Eqs. (3.1) and (3.7) imply $d M=T d S$. For $\nu_{2}=0$ our solution becomes the usual black brane solution with the $\phi \sim \log r$ singularity at $r=0$ [29], and again Eqs. (3.1) and (3.7) entail $d M=T d S$.

Let us now use the constraint (2.14) to express $M, T, S$ in terms of the single parameter $\nu_{2}$, with $0 \leq \nu_{2} \leq 1$. We have

$$
\begin{aligned}
T & =\frac{1}{4 \pi} \frac{3 \gamma}{1+3 \beta^{2}} \nu_{2}^{\frac{3 \beta^{2}-1}{3 \gamma}}\left(1-\nu_{2}^{2}\right)^{1 / 3}, \quad S=4 \Omega \pi \nu_{2}^{-\frac{2}{3 \gamma}}\left(1-\nu_{2}^{2}\right)^{2 / 3}, \\
M & =\frac{2 \Omega}{1+3 \beta^{2}}\left(\frac{1}{\nu_{2}}+\left(2 \beta^{2}-1\right) \nu_{2}\right), \quad d M=\frac{2 \Omega}{1+3 \beta^{2}}\left(-\frac{1}{\nu_{2}^{2}}+\left(2 \beta^{2}-1\right)\right) d \nu_{2}, \\
T d S & =\frac{2 \Omega}{1+3 \beta^{2}}\left(\gamma d \nu_{1}+\beta^{2} \nu_{1} d \log \left(\nu_{1}+\nu_{2}\right)\right)=\frac{2 \Omega}{1+3 \beta^{2}}\left(-\frac{1}{\nu_{2}^{2}}+\left(2 \beta^{2}-1\right)\right) d \nu_{2} .
\end{aligned}
$$

The first principle of thermodynamics $d M=T d S$ is therefore satisfied.

\section{PHASE TRANSITION}

In this section we discuss the thermodynamical properties of our solutions. The thermodynamical behavior of the black brane depends crucially on the value of $\beta$. It is in particular evident from Eq. (3.8) that $\beta^{2}=1 / 3$ is a transition value. We will therefore discuss separately the three cases $\beta^{2}<1 / 3, \beta^{2}=1 / 3, \beta^{2}>1 / 3$. 
A. The $\beta^{2}<1 / 3$ case

For $\beta^{2}<1 / 3$ the scalar black brane exists in the full range of temperatures, $0 \leq T<\infty$, with $\nu_{2}=1,0$ corresponding, respectively to $T=0, \infty$ and to $S=0, \infty$. Notice that the parameter range $\beta^{2}<1 / 3$ corresponds to the region for which the scalar black brane solution has an holographic interpretation and the $r=\infty$ region is timelike.

Let us now compute the free energy $F=M-T S$ for the scalar black brane solution (2.12) and for the SAdS solutions (2.5). For the scalar black brane we get

$$
F_{S B}(T)=\frac{\Omega}{1+3 \beta^{2}}\left(\frac{3 \beta^{2}-1}{\nu_{2}}+\left(\beta^{2}+1\right) \nu_{2}\right),
$$

where $\nu_{2}=\nu_{2}(T)$ is defined implicitly by Eq. (3.8). For the SAdS black brane we simply have

$$
F_{S A d S}(T)=-\Omega\left(\frac{4 \pi}{3}\right)^{3} T^{3} .
$$

It is easy to check using Eqs. (4.1) and (3.8) that $F_{S B}(T)$ is a monotonic decreasing function of $T$.

One can now show that $\Delta F=F_{S B}-F_{S A d S}$ is positive for small $T$, but becomes negative at large $T$. This can be seen by first considering the small- $T\left(\nu_{2} \sim 1\right)$ behavior of $F_{S B}$,

$$
F_{S B}(T)=\Omega\left(\frac{4 \beta^{2}}{3 \beta^{2}+1}-\frac{2(4 \pi)^{3}}{27 \gamma^{2}}\left(3 \beta^{2}+1\right)^{2} T^{3}+\mathcal{O}\left(T^{6}\right)\right) .
$$

Notice that this small- $T$ behavior is dictated by the $T=0, \mathrm{AdS}_{4}$ extremal limit and corresponds to a holographically dual 3D CFT for which the free energy scales as $F \sim T^{3}$. On the other hand the large- $T\left(\nu_{2} \sim 0\right)$ behavior is given by

$$
F_{S B}(T)=\Omega \frac{3 \beta^{2}-1}{3 \beta^{2}+1}\left(\frac{4 \pi\left(3 \beta^{2}+1\right)}{3 \gamma}\right)^{\frac{3 \gamma}{1-3 \beta^{2}}} T^{\frac{3 \gamma}{1-3 \beta^{2}}} .
$$

This large- $T$ scaling behavior of the free energy can be also interpreted in terms of hyperscaling violation in the dual QFT (see Sect. V).

The free energy starts positive at small $T$, with $F(T=0)=\Omega \frac{4 \beta^{2}}{3 \beta^{2}+1}>0$, so that $\Delta F>0$, but, being $3 \gamma /\left(1-3 \beta^{2}\right)>3, \Delta F$ turns negative at large $T$. This implies the existence of a critical temperature $T_{c}$ at which $\Delta F\left(T_{c}\right)=0 . T_{c}$ can be determined graphically and numerically. By equating $F_{S B}=F_{S A d S}$ we get :

$$
g(y)=1-\beta^{2} \frac{3+y}{1-y}=f(y)=\frac{\gamma^{3}}{\left(1+3 \beta^{2}\right)^{2}} y^{\frac{\beta^{2}}{\gamma}},
$$

where $y=\nu_{2}^{2}$.

One can easily realize that the two curves $f(y)$ and $g(y)$ do not intersect for $\beta^{2} \geq 1 / 3$, while they intersect at a finite non-vanishing value of the temperature for $\beta^{2}<1 / 3$.

In figure 2 we show the behavior of the free energy density for a selected value of $\beta\left(\beta^{2}=1 / 4\right)$ in the range $0 \leq \beta^{2}<1 / 3$. For $\beta^{2}=1 / 4$ we have also computed numerically the critical temperature, that results $T_{c}=0.109583$.

It is also of some interest to determine the behavior of $F$ near $T_{c}$, i.e. its cross-over between the two phases. Near $T_{c}, F$ has $\beta$-independent behavior, and scales as

$$
F_{S B} \sim\left(\frac{T_{c}-T}{T_{c}}\right)^{\alpha},
$$

with $\alpha=1 / n$, where the integer $n$ is the order of the first non-vanishing derivative of $T$ evaluated at $T_{c}$. For $\beta^{2}<1 / 3,\left(d T / d \nu_{2}\right)\left(T_{c}\right) \neq 0$ so that $n=1$. 


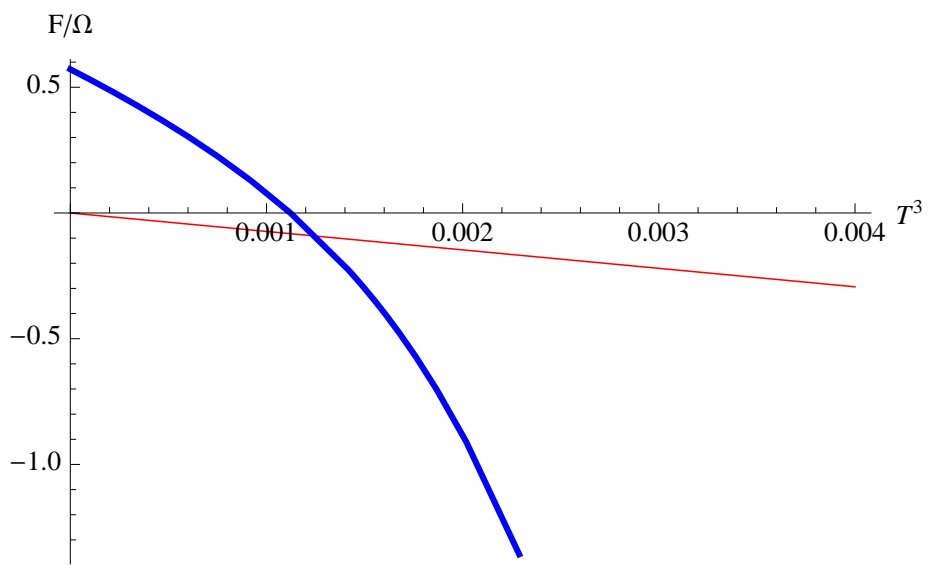

FIG. 2: Plot of the free energy density $F / \Omega$ of the scalar black brane for $\beta^{2}=1 / 4$ (blue, thick line) and of the SAdS black brane (red, thin line) as function of $T^{3}$.

Summarizing, in the case $\beta^{2}<1 / 3$, above the critical temperature $T_{c}$ the free energy $F$ of our black brane solution (2.12) becomes smaller than the free energy of the SAdS solution, and therefore the SAdS solution becomes unstable and the system undergoes a first-order phase transition. The phase transition is first-order because at $T=T_{c}, d F_{S B} / d T \neq d F_{S A d S} / d T$. The first derivative of free energy and the specific heat $(d F / d T)+S$ are therefore discontinuous at $T=T_{c}$. The presence of this phase transition has to be understood as a cross-over from a $T<T_{c}$ regime, in which the AdS solution (2.9) with vanishing scalar field is energetically preferred, to $T>T_{c}$ regime, in which the scale covariant, domain wall solution (2.10) dressed with scalar hair is energetically preferred.

The phase transition found in this section can also be described, holographically, in terms of the dual QFT. The scalar field $\phi$ has to be interpreted in the dual field theory as an order parameter, or equivalently a VEV of a scalar operator $\langle O\rangle$ controlling a phase transition between a phase in which $\langle O\rangle=0$ (on the gravity side, the SAdS phase) and a phase in which $\langle O\rangle \neq 0$ (on the gravity side, the scalar black brane phase endowed with a non trivial scalar field). At small temperature the behavior of the system is determined by the IR fixed point with $\langle O\rangle=0$. At large temperatures, the system is ruled by UV physics, in which the scalar-dressed phase is energetically preferred and develops a large negative $\phi$ (corresponding to large negative values of $\langle O\rangle$ ), with a vanishing self-interaction potential $V(\phi)$.

\section{B. The $\beta^{2}=1 / 3$ case}

For $\beta^{2}=1 / 3$, it is evident from Eq. (3.8) that, since $0 \leq \nu_{2} \leq 1$, the scalar black brane solution exists only for temperatures below a critical temperature $T=T_{c}=1 / 4 \pi$. Above $T_{c}$ only the SAdS solution (2.5) exists. This behavior, namely the existence of scalar-dressed solution only below a critical temperature, has been found, numerically, in several Einstein-Maxwell-scalar models.

For $\beta^{2}=1 / 3$ the free energy $F_{S B}(T)$ in Eq. (4.1) can be written explicitly. We have

$$
F_{S B}=\frac{2 \Omega}{3} \sqrt{1-(4 \pi T)^{3}} .
$$

The free energy is positive definite and vanishes for $T=T_{c}$, whereas $F_{S A d S}$ is always negative. Also in the temperature range $T \leq T_{c}$, where the scalar black brane exists, we have $F_{S A d S}<F_{S B}$ and the SAdS solution is always energetically favored. Although this phase is unstable with respect to the SAdS phase, it is nevertheless interesting to determine the scaling behavior of $F_{S B}$.

Since for $\beta^{2}=1 / 3\left(d T / d \nu_{2}\right)\left(T_{c}\right)=0$ but $\left(d^{2} T / d \nu_{2}^{2}\right)\left(T_{c}\right) \neq 0$, near $T_{c}$ the scaling behavior is 
given by Eq. (4.6) with $n=2$,

$$
F_{S B} \sim\left(\frac{T_{c}-T}{T_{c}}\right)^{1 / 2}
$$

Near $T=0$, which corresponds to the $\mathrm{AdS}_{4}$ spacetime, we have the expected scaling behavior of a CFT in $2+1$ dimensions:

$$
F_{S B}=\frac{2 \Omega}{3}\left(1-\frac{(4 \pi T)^{3}}{2}\right)
$$

\section{The $\beta^{2}>1 / 3$ case}

For $\beta^{2}>1 / 3$ the function $T\left(\nu_{2}\right)$ of Eq. (3.8) is not monotonic, but in the range of definition of $\nu_{2}, \quad 0 \leq \nu_{2} \leq 1$ it has a local maximum at $\nu_{2}=\nu_{0}=\sqrt{\left(3 \beta^{2}-1\right) /\left(\beta^{2}+1\right)}$. This means that also in this case the scalar black brane solution exists only below a critical temperature $T_{c} \equiv T\left(\nu_{0}\right)=3 \gamma^{4 / 3}\left[2^{5 / 3}\left(3 \beta^{2}-1\right)^{\left(1-3 \beta^{2}\right) / 6 \gamma} \pi\left(1+3 \beta^{2}\right)\left(1+\beta^{2}\right)^{\left(1+\beta^{2}\right) / 6 \gamma}\right]^{-1}$. Notice that $F$ has a minimum in $\nu_{0}$. In figure 3 we show the function $T\left(\nu_{2}\right)$ for $\beta^{2}=2 / 3$. In this case $\nu_{0}=\sqrt{3 / 5}$ and $T_{c}=2^{1 / 3} 5^{-5 / 6} /(\sqrt{3} 4 \pi)$.

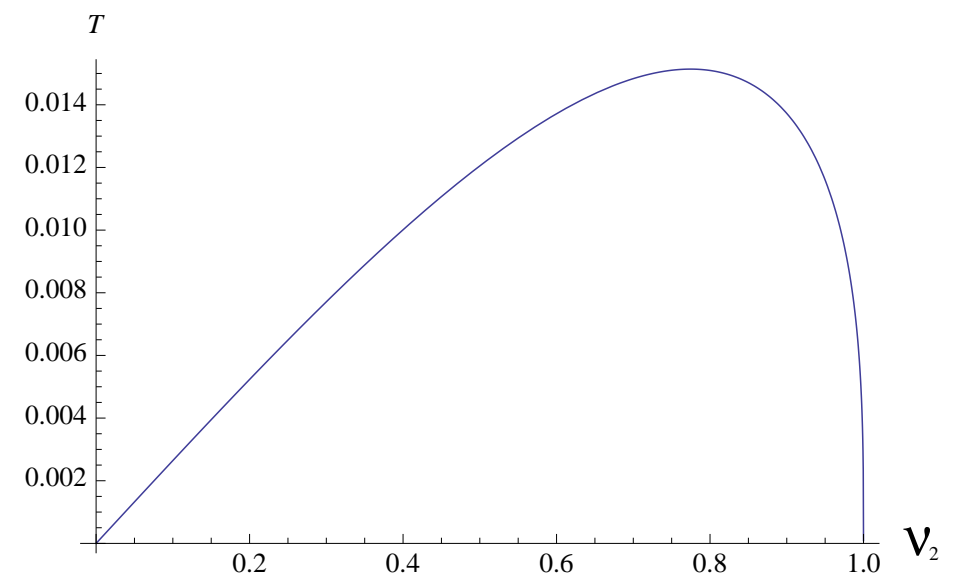

FIG. 3: Plot of the function $T\left(\nu_{2}\right)$ for $\beta^{2}=2 / 3$.

For $T \leq T_{c}$, our model admits both the scalar black brane and the SAdS solutions. Above $T_{c}$, only the SAdS solution exists. Differently from the $\beta^{2}=1 / 3$ case, the non-monotonicity of $T\left(\nu_{2}\right)$ implies the existence of two different branches of the scalar-dressed black brane phase for $T \leq T_{c}$. From Eq. (4.1) one easily realizes that for $\beta^{2}>1 / 3, F_{S B}$ is always positive, and hence $F_{S B}>F_{S A d S}$, implying instability of the two phases with respect to the SAdS phase. The behavior of the free energy density for the two branches is shown in Fig. 团 for $\beta^{2}=2 / 3$.

Even if they are unstable, it is however of interest to briefly discuss the features of these small- $T$ phases. The first branch is obtained for $\nu_{0} \leq \nu_{2}<1$ and is the usual $\mathrm{AdS}_{4}$ phase we have already obtained at small $T$ when $\beta^{2} \leq 1 / 3$. As expected, in this case the free energy scales for $T \sim T_{c}$ and $T \sim 0$ as in Eqs. (4.8) and (4.9), respectively.

The second branch is obtained for $0 \leq \nu_{2} \leq \nu_{0}$ and has no analogue for $\beta^{2} \leq 1 / 3$. The free energy scales at small temperatures as in Eq. (4.4), $F \sim T^{\alpha}, \alpha=3 \gamma /\left(1-3 \beta^{2}\right)$. But now $\alpha$ is negative and we have a singularity of $F_{S B}$ at $T=0$. This small- $T$ singular behavior is due to the fact that for $\nu_{2}=0$ there is no $T=0$ extremal soliton, but an extremal domain wall solution with the scalar behaving as $\log r$.

Near $T_{c}$ the scaling behavior of $F_{S B}$ is the same as in the $\beta^{2}=1 / 3$ case. It is given by Eq. (4.8). 


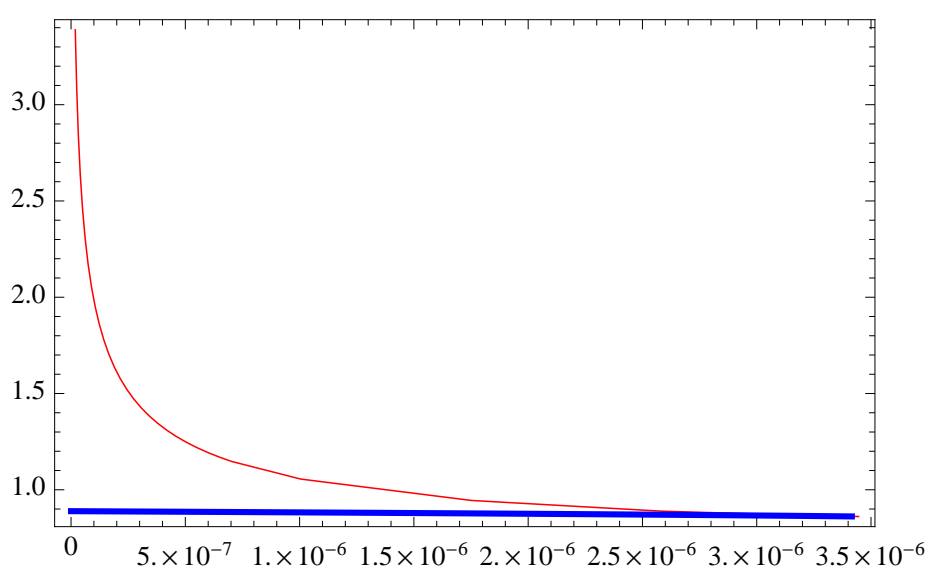

FIG. 4: Plot of the free energy density $F_{S B} / \Omega$ as a function of $T^{3}$ for the two branches of the solution in the $\beta^{2}=2 / 3$ case. The blue (thick) line indicates the first $\left(\mathrm{AdS}_{4}\right)$ branch, whereas the red (thin) line indicates the second branch.

\section{HYPERSCALING VIOLATION}

The different thermodynamical phases of our scalar black brane and their peculiar behavior described in the previous sections can also be understood in terms of its symmetries in the UV and IR regimes.

In particular, this thermodynamical pattern finds its natural explanation in terms of the UV hyperscaling violation generated in the dual QFT by the asymptotic DW solution (2.10) with respect to the IR scaling-preserving solution given by the extremal $\mathrm{AdS}_{4}$ spacetime (2.9). In fact, the hyperscaling-violating phase is associated with the emergence of an UV length scale $r_{0}$, which obviously decouples in the IR conformal phase.

This behavior is peculiar and somehow unexpected, because the regime of the dual QFT in which one naturally expects hyperscaling violation to occur is not the UV but the IR. This is not only true for the condensed matter systems for which hyperscaling violation was originally discovered [37], but also for its recent holographic realizations [19, 20, 36].

The description of holographic hyperscaling violation in $d+2$ dimensions is based on the scale covariant metric $[36]^{3}$

$$
d s^{2}=\frac{1}{r^{2}}\left(-\frac{d t^{2}}{r^{2 d(z-1) /(d-\theta)}}+d x_{i} d x_{i}+r^{2 \theta /(d-\theta)} d r^{2}\right)
$$

where $d$ is the number of transverse dimensions, $\theta$ is the hyperscaling violation parameter and $z$ is the dynamic critical exponent (it describes anisotropic scaling, hence violation of Poincaré symmetry, in the $d+1$ spacetime). The transformation law under rescaling of the coordinates is

$$
t \rightarrow \lambda^{z} t, \quad x_{i} \rightarrow \lambda x_{i}, \quad r \rightarrow \lambda^{(d-\theta) / d} r, \quad d s \rightarrow \lambda^{\theta / d} d s .
$$

For $\theta=0$ the metric (5.1) is scale invariant, hence for generic values of $z$ it gives the Lifshitz spacetime. For $z=1$ the $d+1$-dimensional spacetime has Poincaré isometry. Finally, for $\theta=0$,

\footnotetext{
${ }^{3}$ In the literature there are several, equivalent, definitions of scale covariant metrics generating in the dual QFT hyperscaling violation. They are related by reparametrizations of the radial coordinate $r$. Here, we use the metric of reference [36], but the same results can be obtained using for instance the metric of reference [20].
} 
$z=1$, the metric (5.1) becomes the $\operatorname{AdS}_{d+2}$ spacetime with both Poincaré isometry in the $d+1$ sections and scaling (conformal) symmetry. Taking into account that the the temperature scales as an inverse time, in presence of a black brane the area law together with Eq. (5.2) implies the following scaling behavior for the free energy:

$$
F \sim T^{\frac{(d-\theta)+z}{z}} .
$$

This relation allows a simple physical interpretation of the hyperscaling violation parameter $\theta$, which is analogous to that used in condensed matter critical system: the hyperscaling relation between specific heat $\hat{\alpha}$ and critical exponent $\hat{\nu}$, given by $2-\hat{\alpha}=d \hat{\nu}$ is modified by "lowering" the dimensionality of the system from $d$ to $d-\theta$, namely $2-\hat{\alpha}=(d-\theta) \hat{\nu}$.

The $r=\infty$ asymptotic form of our scalar black brane solutions (2.10) (we set here $r_{0}=1$ ) can be brought in the form (5.1) by a redefinition of the radial coordinate,

$$
r \rightarrow r^{-\left(1+3 \beta^{2}\right)} .
$$

Notice that using the radial coordinate of the metric (5.1) the UV region of the dual QFT is described by $r=0$, whereas the IR region corresponds to $r=\infty$. Consistently, with Eq. (5.4) the role played by $r=0$ and $r=\infty$ is reversed when one uses the radial coordinate of the metric (2.10).

Taking into account that in our case $d=2$, we easily find that the asymptotic black brane metric (2.10) is characterized by the following parameters:

$$
z=1, \quad \theta=\frac{6 \beta^{2}}{3 \beta^{2}-1} .
$$

On the other hand the values of the parameters $z$ and $\theta$ for the extremal $\mathrm{AdS}_{4}$ solution (2.9) are obviously given by

$$
z=1, \quad \theta=0 .
$$

The value $z=1$, implying Poincaré isometry in the $(d+1)$-dimensional spacetime, is largely expected for a scalar, electromagnetically neutral, solution. In fact it is well known that in order to obtain $z \neq 1$ (Lifshitz) solutions, the brane must carry $U(1)$ charge. Inserting in Eq. (5.3) $z=1$, $d=2$, we get

$$
F \sim T^{3-\theta} .
$$

As a check of the correctness of our result, one can recover Eqs. (4.4) and (4.3) inserting, respectively, Eqs. (5.5) and (5.6) into Eq. (5.7).

As expected, we have $\theta \neq 0$ in the scalar black brane phase. This gives the deviation from the conformal scaling of the free energy of a $2+1$ conformal field theory, which one obtains for $\theta=0$, in the hyperscaling-preserving phase.

On the other hand, the particular values (5.5) of the hyperscaling-violation parameter for our model are rather intriguing. The parameter $\theta$ is negative for $\beta^{2}<1 / 3$, diverges for $\beta^{2}=1 / 3$ and becomes positive, with $\theta \geq 3$, for $\beta^{2}>1 / 3$. Although $\theta$ is negative, one can easily realize that the null energy conditions for the bulk stress-energy tensor are satisfied. In fact for $z=1$ these conditions require either $\theta \leq 0$ or $\theta \geq d[20]$.

The negative value of $\theta$ for $\beta^{2}<1 / 3$ has no counterpart in condensed matter critical system, for which the hyperscaling-violating parameter is positive. This is probably related to another striking difference between the two cases. In the condensed matter case the hyperscaling-violating phase is stable at small temperatures where (for instance random-field induced) long-scale fluctuations dominate over thermal fluctuation. In our gravitational-holographic case the opposite happens. The hyperscaling-violating phase is stable at large temperatures, but becomes unstable at small temperatures, where the hyperscaling-preserving phase is energetically preferred. This interchange of IR and UV physics is a rather puzzling point, whose physical meaning is presently not clear to 
us. It is possible that negative values of $\theta$ are related to the fact that our gravitational system at large temperatures seems to prefer to live in more than $3+1$ dimensions. This possibility is also supported by the fact that our model arises from compactifications of black $(p-1)$-branes [10]. Moreover, negative values of $\theta$ have been obtained in some string theory constructions based on Dp branes 20]

Naively, one could hope that the behavior in the $\beta^{2}>1 / 3$ case would shed light on these puzzling features. Although the hyperscaling-violating phase (the second branch discussed in Subsection IVCD is in this case unstable, nevertheless it occurs at small temperatures, is characterized by a positive $\theta$ and the null energy condition $\theta>d$ [20] for the bulk stress-energy tensor is satisfied. Unfortunately, in this case $\theta$ is greater than 3, so that the free energy (5.7) scales at small temperatures with a negative exponent (see also Eq. (4.4)) and becomes singular at $T=0$.

\section{THE $\beta^{2}>1$ CASE}

The solutions for $\beta^{2}>1$ have also been obtained in [1]. Actually, due to the invariance of the action for $\beta \rightarrow 1 / \beta$, they can simply be recovered from (2.6) by duality, substituting everywhere $\beta$ with $1 / \beta$. Note that for $\beta^{2}>1$ the parameter $\gamma$ becomes negative.

The solutions can be written as

$$
\begin{aligned}
d s^{2} & =\Delta(r)^{-\frac{2}{3 \gamma}}\left[\left(\frac{r}{r_{0}}\right)^{\frac{2 \beta^{2}}{3+\beta^{2}}}\left(-\Gamma(r) d t^{2}+d x_{i} d x_{i}\right)+E \frac{\Delta(r)^{-\frac{4}{3 \gamma}}}{\Gamma(r)}\left(\frac{r}{r_{0}}\right)^{-\frac{2 \beta^{2}}{3+\beta^{2}}} d r^{2}\right], \\
e^{2 \phi} & =\left[\frac{\Delta(r)}{D}\left(\frac{r}{r_{0}}\right)^{-\frac{3 \gamma}{3+\beta^{2}}}\right]^{\frac{2 \beta}{\sqrt{3} \gamma}},
\end{aligned}
$$

where $\Gamma(r)=1-\mu_{1}\left(\frac{r_{0}}{r}\right)^{\delta}, \Delta(r)=1+\mu_{2}\left(\frac{r_{0}}{r}\right)^{\delta}, \delta=-3 \gamma /\left(3+\beta^{2}\right), D=\sqrt{\mu_{2}\left(\mu_{1}+\mu_{2}\right)}, E=$ $\left(\frac{\gamma L}{\left(3+\beta^{2}\right) r_{0}}\right)^{2} D^{2 / \gamma}$, and $\mu_{1} \geq 0, \mu_{2} \geq 0$ are free parameters.

For $r \rightarrow \infty$, the solutions behave as domain walls

$$
d s^{2}=r^{\frac{2 \beta^{2}}{3+\beta^{2}}}\left(-d t^{2}+d x_{i} d x_{i}\right)+r^{-\frac{2 \beta^{2}}{3+\beta^{2}}} d r^{2} .
$$

with $\phi=-\left[(\sqrt{3} \beta) /\left(3+\beta^{2}\right)\right] \log r$.

The properties of the the metric (6.1) are analogous to those holding in the $\beta^{2}<1$ case. If $\mu_{2} \geq 0$, it exhibits a singularity at $r=0$, shielded by a horizon at $\frac{r}{r_{0}}=\mu_{1}^{1 / \delta}$, and therefore represents a regular black brane.

For $\mu_{2}>0$, the extremal limit $\mu_{1}=0$, of solution (6.1) represents a regular soliton with the same behavior as that described in the $\beta^{2}<1$ case.

The solution can be written in a simpler form by rescaling the coordinates and defining new parameters $\nu_{1}$ and $\nu_{2}$, as in the $\beta^{2}<1$ case:

$$
\begin{aligned}
& d s^{2}=\left(\frac{\gamma L}{3+\beta^{2}}\right)^{2}\left[-\Delta(r)^{-\frac{2}{3 \gamma}}\left(1-\frac{\nu_{1}}{r^{\delta}}\right) r^{\frac{2 \beta^{2}}{3+\beta^{2}}} d t^{2}+\frac{\Delta(r)^{-\frac{2}{\gamma}} d r^{2}}{\left(1-\frac{\nu_{1}}{r^{\delta}}\right) r^{\frac{2 \beta^{2}}{3+\beta^{2}}}}\right]+\Delta(r)^{-\frac{2}{3 \gamma}} r^{\frac{2 \beta^{2}}{3+\beta^{2}}} d x_{i} d x_{i}, \\
& e^{2 \phi}=\Delta(r)^{\frac{2 \beta}{\sqrt{3} \gamma}} r^{-\frac{2 \sqrt{3} \beta}{3+\beta^{2}}} .
\end{aligned}
$$

The new parameters are not independent, but satisfy the relation (2.14). Thus, in this form the solution has only one independent parameter, $\nu_{2}$, with $0<\nu_{2} \leq 1$.

The thermodynamics of the $\beta^{2}>1$ solutions can be obtained repeating the calculations of sect. III) or simply by duality from the case $\beta^{2}<1$. 
For the temperature $T$ and entropy $S$ of the solution (6.3) we get

$$
T=-\frac{1}{4 \pi} \frac{3 \gamma}{3+\beta^{2}}\left(\nu_{1}+\nu_{2}\right)^{\frac{2}{3 \gamma}} \nu_{1}^{1 / 3}, \quad S=4 \pi \Omega\left(\nu_{1}+\nu_{2}\right)^{-\frac{2}{3 \gamma}} \nu_{1}^{2 / 3}
$$

while the mass is

$$
M=\frac{2 \Omega}{3+\beta^{2}}\left(\beta^{2} \nu_{1}+2 \nu_{2}\right) .
$$

We can now use the constraint (2.14) to express $M, T, S$ in terms of the independent parameter $\nu_{2}$. They read

$$
M=\frac{2 \Omega}{3+\beta^{2}}\left(\frac{\beta^{2}}{\nu_{2}}+\left(2-\beta^{2}\right) \nu_{2}\right), \quad T=\frac{1}{4 \pi} \frac{-3 \gamma}{3+\beta^{2}} \nu_{2}^{\frac{\beta^{2}-3}{3 \gamma}}\left(1-\nu_{2}^{2}\right)^{1 / 3}, \quad S=4 \Omega \pi \nu_{2}^{\frac{2 \beta^{2}}{3 \gamma}}\left(1-\nu_{2}^{2}\right)^{2 / 3},
$$

It is easy to check that the first principle of thermodynamics $d M=T d S$ is satisfied.

Finally, the free energy $F=M-T S$ for the black brane solution (6.3) is given by

$$
F_{S B}(T)=\frac{\Omega}{3+\beta^{2}}\left(\frac{3-\beta^{2}}{\nu_{2}}+\left(1+\beta^{2}\right) \nu_{2}\right),
$$

where $\nu_{2}=\nu_{2}(T)$ is defined implicitly by Eq. (6.4).

The thermodynamical properties of the solutions with $\beta^{2}>1$ follow from the previous discussion of the $\beta^{2}<1$ case by duality. In particular, one must distinguish three cases, $\beta^{2}>3, \beta^{2}=3, \beta^{2}<$ 3 . We summarize their properties.

\section{A. The $\beta^{2}>3$ case}

This case is analogous to $\beta^{2}<1 / 3$. For $\beta^{2}>3$, the temperature of the black brane can range from 0 to $\infty$. It is easy to check using Eqs. (6.4) and (6.7) that $F_{S B}(T)$ is a monotonic decreasing function of $T$. Moreover, $\Delta F=F_{S B}-F_{S A d S}$ is positive for small $T$ but becomes negative at large $T$. In fact, the small- $T$ behavior of $F_{S B}$ is

$$
F_{S B}(T)=\Omega\left(\frac{4}{3+\beta^{2}}-\frac{(4 \pi)^{3}}{27 \gamma^{2}}\left(3+\beta^{2}\right)^{2} T^{3}+\mathcal{O}\left(T^{6}\right)\right),
$$

while the large- $T$ behavior is given by

$$
F_{S B}(T)=\Omega \frac{3-\beta^{2}}{3+\beta^{2}}\left(\frac{4 \pi\left(\beta^{2}+3\right)}{-3 \gamma}\right)^{-\frac{3 \gamma}{\beta^{2}-3}} T^{-\frac{3 \gamma}{\beta^{2}-3}} .
$$

Hence, $\Delta F$ is positive for $T$ small, but becomes negative at large $T$. This implies the existence of a critical temperature $T_{c}$ at which $\Delta F\left(T_{c}\right)=0$.

Therefore, when $\beta^{2}>3$, the free energy $F$ of the black brane solution (6.3) becomes smaller than the free energy of the Schwarzschild-anti de Sitter (SAdS) solution above the critical temperature $T_{c}$, and hence the SAdS solution becomes unstable and the system undergoes a first-order phase transition.

Analogously to $\beta^{2}<1$ also in the $\beta^{2}>1$ case, we can characterize the solutions with the dynamic critical exponent $z$ and the hyperscaling-violation parameter $\theta$. We have

$$
z=1, \quad \theta=\frac{6}{3-\beta^{2}}
$$




\section{B. The $\beta^{2}=3$ case}

It is evident from Eq. (6.4) that for $\beta^{2}=3$, the temperature of the black brane solution is always lower than a critical temperature $T_{c}=1 / 4 \pi$. Above $T_{c}$ only the SAdS solution (2.5) exists.

For $\beta^{2}=3$, the free energy $F_{S B}(T)$ can be written explicitly, and is equal to that of the $\beta^{2}=1 / 3$ black brane (4.7). The thermodynamics of the $\beta^{2}=3$ black brane is therefore identical to that discussed in the $\beta^{2}=1 / 3$ case.

\section{The $\beta^{2}<3$ case}

As for $1 / 3<\beta^{2}<1$, also for $1<\beta^{2}<3$ the function $T\left(\nu_{2}\right)$ is not monotonic, but has a local maximum, located at $\nu_{0}=\sqrt{\left(3-\beta^{2}\right) /\left(1+\beta^{2}\right)}$. Therefore, also in this case the temperature of the scalar black brane solution must be less than a critical temperature $T_{c} \equiv T\left(\nu_{0}\right)=3 \gamma^{4 / 3}(3-$ $\left.\beta^{2}\right)^{\left(\beta^{2}-3\right) / 6 \gamma}\left(1+\beta^{2}\right)^{\left(1+\beta^{2}\right) / 6 \gamma}\left[2^{5 / 3} \pi\left(3+\beta^{2}\right)\right]^{-1}$.

For $T \leq T_{c}$ our model admits both the scalar black brane and the SAdS solutions. Above $T_{c}$ only the SAdS solution exists. The non-monotonicity of $T\left(\nu_{2}\right)$ implies the existence of two different branches of the scalar-dressed black brane phase for $T \leq T_{c}$. Since in the present case $F_{S B}$ is always positive, we necessarily have $F_{S B}>F_{S A d S}$, implying instability of the two phases with respect to the SAdS phase.

The first branch is obtained for $\nu_{0} \leq \nu_{2}<1$ and is the usual $\mathrm{AdS}_{4}$ phase. As expected, the free energy scales for $T \sim T_{c}$ and $T \sim 0$ as in Eqs. (6.8) and (6.9), respectively. The second branch is obtained for $0 \leq \nu_{2} \leq \nu_{0}$. In this case, the free energy scales at small temperatures as in Eq. (6.9), $F \sim T^{\alpha}, \alpha=3 \gamma /\left(3-\beta^{2}\right)$, but now $\alpha$ is negative and we have a singularity of $F_{S B}$ at $T=0$.

\section{GENERAL MODELS AND CHARGED SOLUTIONS}

Until now we have restricted our investigation to the uncharged case and the particular model described by the potential (2.3). In this section we will extend our discussion to general models described by Eq. (2.2) and to charged solutions.

\section{A. General models}

The main features of the black brane solutions described in the previous sections are not a peculiarity of the model (2.3) but are determined by the behavior of the potential (2.3) at $\phi=0$ and $\phi=-\infty$ (see Eqs. (2.2)).

In Ref. 29] has been derived the general asymptotic solution of a model with an exponential potential,

$$
\begin{aligned}
V(\phi) & =-\frac{2\left(3-h^{2}\right)}{\left(1+h^{2}\right)^{2}} e^{2 h \phi}, \quad \phi=-\frac{h}{h^{2}+1} \log r \\
d s^{2} & =r^{\frac{2}{h^{2}+1}}\left(-d t^{2}+d x_{i} d x_{i}\right)+r^{-\frac{2}{h^{2}+1}} d r^{2} .
\end{aligned}
$$

The case described by Eq. (2.2) is covered by setting $h^{2}<3$ in Eq. (7.1), so that the $r=\infty$ region corresponds to $\phi=-\infty$ where the potential approaches zero exponentially. On the other hand the condition (2.2) at $\phi=0$ implies the existence of an $\mathrm{AdS}_{4}$ vacuum for $\phi=0$.

Obviously, a generic model will not be exactly integrable. The existence of a scalar black brane solution interpolating between the $\mathrm{AdS}_{4}$ vacuum at $r=0$ and the scale covariant solution (7.1) at $r=\infty$ can only be proved numerically. Nevertheless, if such a solution exists, then necessarily the thermodynamical system for $h^{2}<1$ must undergo the scalar black brane $\rightarrow$ SAdS phase transition described in Sect. IV. 
This can be shown by first realizing that at small $T$ the free energy of the scalar black brane must have a behavior similar to that of Eq. (4.3), i.e. $F_{S B}=C_{1}-C_{2} T^{3}$, with $C_{1,2}$ positive constants. This implies that at small $T, F_{S B}-F_{S A d S}>0$. On the other hand at large $T$ the entropy of a scalar black brane with the asymptotic behavior (7.1) scales as $S \sim T^{2 /\left(1-h^{2}\right)}$, implying the scaling for the free energy $F_{S B} \sim-T^{\left(3-h^{2}\right) /\left(1-h^{2}\right)}$. For $h^{2}<1$ we have $T^{\left(3-h^{2}\right) /\left(1-h^{2}\right)}>T^{3}$, from which follows that at large $T, F_{S B}-F_{S A d S}<0$. This proves the existence of a scalar black brane $\rightarrow$ SAdS phase transition and of an associated critical temperature.

Comparing equation (7.1) with Eq. (5.1) one can read off, using a suitable reparametrization of the radial coordinate, the hyperscaling violation parameter $\theta=2 h^{2} /\left(h^{2}-1\right)$, and the dynamic critical exponent $z=1$. Notice that $\theta$ is negative for $h^{2}<1$.

Comparison of the large- $T$ behavior of our models with that characterizing the small- $T$ regime of the usual models for holographic hyperscaling violation sheds some light on the nature of the phase transition. The latter models use potentials $V(\phi)$ that at $\phi=0$ have a maximum instead of a minimum. This corresponds to a local tachyonic scalar excitation near $\phi=0$ with mass slightly above the BF bound. For $\phi \rightarrow \infty$, the potential diverges exponentially. This $\phi \rightarrow \infty$ regime is also described by Eq. (7.1) with $h^{2}<3$, but now $\phi \rightarrow \infty$ corresponds to the IR region $r=0$, whereas $\phi=0$ describes the $r=\infty$ UV region. In some sense the phase transition described in this paper is an UV counterpart of the IR phase transition of usual hyperscaling-violating models.

\section{B. Charged solution}

Let us now briefly discuss the electrically charged case. A Maxwell field can be introduced in the action (2.1) in two different ways, that is with minimal or non-minimal coupling to the scalar field. In the minimal case the action (2.1) becomes

$$
I=\int d^{4} x \sqrt{-g}\left[R-2(\partial \phi)^{2}-F^{2}-V(\phi)\right] .
$$

Let us briefly discuss a model with a potential $V$ given by Eq. (2.3). Needless to say, our considerations can be trivially extended to a generic potential satisfying the conditions (2.2).

For $\phi=$ const. $=0$, the model admits the Reissner-Nordstrom-anti de Sitter (RNAdS) solution. For non-trivial scalar field configurations, the exact integrability valid in the neutral case is lost, but one can treat the problem using the same method described in the previous sections.

In the asymptotic region $r \rightarrow \infty, \phi \rightarrow-\infty$ the potential behaves exponentially

$$
V(\phi)=-\frac{6 \beta^{2}}{\gamma L} e^{2 \sqrt{3} \beta \phi} .
$$

In the charged case the asymptotic form of the solution is [29],

$$
d s^{2}=-r^{\frac{8-6 \beta^{2}}{4+3 \beta^{2}}} d t^{2}+r^{-\frac{8-6 \beta^{2}}{4+3 \beta^{2}}} d r^{2}+Q r^{\frac{6 \beta^{2}}{4+3 \beta^{2}}} d x_{i} d x_{i}, \quad \phi=-\frac{2 \sqrt{3} \beta}{6 \beta^{2}+4} \log r,
$$

where $Q$ is the electric charge. In the electrically charged case, the small- $T$, small- $r$ behavior is more involved than in the uncharged case. This is mainly because in presence of the electric charge beside the $\mathrm{AdS}_{4}$ vacuum also $\mathrm{AdS}_{2} \times R^{2}$ vacua are possible.

We will not enter into the details, but will just assume the existence of scalar, electrically charged BB solution with asymptotic behavior given by Eq. (7.4), which interpolates between the $T=0$ vacuum and the asymptotic solution (7.4). Typically the existence of this solution has to be shown numerically. The asymptotic solution (7.4) can be put in the form (5.1) by the coordinate transformation $r \rightarrow r^{-\left(4+3 \beta^{2}\right) / 3 \beta^{2}}$. The parameters $z, \theta$ can be easily calculated. We have $\theta=4$, $z=3-4 /\left(3 \beta^{2}\right)$. Notice that in the electrically charged case the hyperscaling violation parameter is $\beta$-independent, whereas it is the dynamic critical exponent $z$ that becomes $\beta$-dependent. Moreover, 
the constraints coming from the null energy condition for the bulk stress-energy tensor (see Ref. [20]) are satisfied for $\beta^{2} \leq 2 / 3$.

With these values of $z$ and $\theta$ Eq. (5.3) gives the large- $T$ behavior of the free energy

$$
F_{S B} \sim-T^{1-\frac{2}{z}}=-T^{\frac{3 \beta^{2}-4}{9 \beta^{2}-4}} .
$$

The scaling exponent for $F$ becomes greater than 3 for $\beta^{2}>1 / 3$. It follows that at large $T$ the free energy of the scalar BB becomes smaller than the free energy of the RNAdS solution. At large $T$ a phase transition scalar $\mathrm{BB} \rightarrow$ RNAdS solution becomes possible. Obviously, the phase diagram of our solution at small $T$ could be rather complicated. The existence of the phase transition has therefore to be confirmed by explicit numerical calculations.

To conclude, let us briefly comment on the case of non-minimal couplings between the Maxwell and the scalar field. The action is now,

$$
I=\int d^{4} x \sqrt{-g}\left[R-2(\partial \phi)^{2}-f(\phi) F^{2}-V(\phi)\right],
$$

where $f(\phi)$ is chosen as a combination of exponentials. These models are of particular relevance because they represent the framework in which holographic hyperscaling violation first emerged [7, 9, 11, 23, 24].

Depending on the properties of the potential $V$ and of the coupling function $f(\phi)$ the model can have an hyperscaling violating phase either in the IR or in the UV. When the potential has a maximum at $\phi=0$, the scalar field $\phi$ behaves as $\phi \sim-\log r$ for $r=0$, in the IR region we have $\phi \rightarrow \infty$ and the coupling function behaves as $f(\phi) \sim e^{\delta \phi}$ we get hyperscaling violation in the IR 7 9, 11, 20, 23, 24]. Conversely, when the potential has a maximum at $\phi=0$ (like, e.g in Eq. (2.3) $), \phi$ behaves as $\phi \sim-\log r$ for $r=\infty$, in the UV region we have $\phi \rightarrow-\infty$ and the coupling function behaves as $f(\phi) \sim e^{\delta \phi}$ we get the hyperscaling violation in the UV discussed in this paper.

\section{CONCLUSIONS}

In this paper we have investigated the thermodynamical behavior and the scaling symmetries of the BB solutions of AdS Einstein-scalar gravity models for which the squared mass for the scalar is positive and the potential vanishes exponentially as $\phi \rightarrow-\infty$.

Our investigation has been mainly focused on an integrable model, which also arises as compactification of black $(p-1)$-branes, for which exact analytic solutions can be found. However, we have been able to show that the relevant features of this model can easily be extend to a broad class of Einstein-scalar and Einstein-Maxwell-scalar gravity models.

We have found that this broad class of models has an interesting thermodynamical phase diagram and extremely non-trivial behavior in the ultraviolet regime of the holographically dual QFT. Hyperscaling violation generates an UV length scale, related to the size of the brane, which decouples in the IR where conformal invariance is restored. At high temperatures the SAdS solution becomes unstable and a scalar-dressed BB solution, with non-AdS, scale-covariant asymptotical behavior, becomes energetically preferred. This new, scalar-dressed phase can be characterized by the two parameters normally used for critical systems with hyperscaling violation: the dynamical critical exponent $z$ and the hyperscaling violation parameter $\theta$.

The actual value of the parameters $z$ and $\theta$ depends on the particular model under consideration, for instance $z=1$ for electrically uncharged $\mathrm{BB}$ and $z \neq 1$ for electrically charged BB. Moreover, quite generically the values of $z, \theta$ satisfies the constraints coming from the null energy conditions [20].

On the other hand the most striking feature of our models is that for uncharged black brane the hyperscaling parameter $\theta$ is always negative.

This is a rather puzzling feature, whose full meaning is presently not clear to us. To our knowledge negative values of $\theta$ are not realized in usual condensed matter critical systems. In the 
holographic framework, negative values of $\theta$ have been obtained in some string theory constructions based on Dp branes [20].

In the usual scenario for holographic hyperscaling violation, when the hyperscaling violation phase is stable in the IR, $\theta$ is positive. This implies that the scaling law for the free energy is that pertinent to a CFT in $d-\theta$ dimensions. This lowering of the "effective dimensions" is a crucial ingredient in the small temperature behavior of traditional (e.g. Ising models) hyperscalingviolating critical systems [37]. The same effect is also a key concept in the holographic explanation of phenomena like hidden Fermi surfaces or the area law violation for entanglement entropy 20, 36, 38, 39]. Conversely, the gravitational models investigated in this paper have a negative hyperscaling-violation parameter $\theta$ and are therefore characterized by a raising of the "effective dimensions". For this reason it does not seem that they could be relevant for applications to condensed matter systems. It is more likely that they could be useful for understanding the holographic features of the gravitational interaction.

A possible way to shed light on the UV behavior of our model is to calculate the correlation functions for the scalar operators in the dual field theory. For positive $\theta$ these correlation functions have been calculated in Ref. 20]. They are characterized by an intriguing cross-over behavior between an exponential form at large distance and an universal, power-law form at short distances. Unfortunately, the short-distance calculations of Ref. [20] do not hold for negative $\theta$, which is the relevant case for our models.

Apart from its interesting ultraviolet features, our model could also be useful for resolving IR singularities of scale covariant metrics. In fact it is known that this kind of metrics are singular at small radius [31, 45, 46]. The restoration of the conformal invariance and the emergence of AdS geometry near $r=0$ realized by our scalar soliton (2.8) represent a natural way to cure the small radius singularities of metrics of the form (2.10).

In this paper we have considered 4-dimensional Einstein-scalar gravity models, however we expect that our results can be easily extended to generic spacetime dimensions. In particular, we expect that the higher-dimensional generalization of our models and solutions will match the general expressions for compactified black $(p-1)$-branes with a smeared charge supported by a $G_{(p+1)}$ field-strength form obtained in Ref. [10].

\section{Acknowledgments}

We thank Blaise Goutéraux and Elias Kiritsis for having pointed out to us that the model and

the solutions discussed in this paper also arise as compactification of black $(p-1)$-branes with a smeared charge.

[1] M. Cadoni, S. Mignemi, and M. Serra, Phys.Rev. D85, 086001 (2012), arXiv:1111.6581.

[2] S. A. Hartnoll, C. P. Herzog, and G. T. Horowitz, Phys. Rev. Lett. 101, 031601 (2008), arXiv:0803.3295.

[3] S. A. Hartnoll, C. P. Herzog, and G. T. Horowitz, JHEP 12, 015 (2008), arXiv:0810.1563.

[4] G. T. Horowitz and M. M. Roberts, Phys.Rev. D78, 126008 (2008), arXiv:0810.1077.

[5] C. P. Herzog, J. Phys. A42, 343001 (2009), arXiv:0904.1975.

[6] S. A. Hartnoll, Class.Quant.Grav. 26, 224002 (2009), arXiv:0903.3246.

[7] C. Charmousis, B. Gouteraux, and J. Soda, Phys. Rev. D80, 024028 (2009), arXiv:0905.3337.

[8] M. Cadoni, G. D’Appollonio, and P. Pani, JHEP 03, 100 (2010), arXiv:0912.3520.

[9] K. Goldstein, S. Kachru, S. Prakash, and S. P. Trivedi, JHEP 1008, 078 (2010), arXiv:0911.3586.

[10] B. Gouteraux and E. Kiritsis, JHEP 1112, 036 (2011), arXiv:1107.2116.

[11] C. Charmousis, B. Gouteraux, B. S. Kim, E. Kiritsis, and R. Meyer, JHEP 11, 151 (2010), arXiv:1005.4690.

[12] G. Bertoldi, B. A. Burrington, and A. W. Peet (2010), arXiv:1007.1464.

[13] M. Cadoni and P. Pani, JHEP 1104, 049 (2011), arXiv:1102.3820. 
[14] B.-H. Lee, D.-W. Pang, and C. Park, Int.J.Mod.Phys. A26, 2279 (2011), arXiv:1107.5822.

[15] M. Fujita, M. Kaminski, and A. Karch (2012), arXiv:1204.0012.

[16] K. Goldstein, N. Iizuka, S. Kachru, S. Prakash, S. P. Trivedi, et al., JHEP 1010, 027 (2010), arXiv:1007.2490.

[17] G. Bertoldi, B. A. Burrington, A. W. Peet, and I. G. Zadeh, Phys.Rev. D83, 126006 (2011), arXiv:1101.1980.

[18] N. Iizuka, N. Kundu, P. Narayan, and S. P. Trivedi, JHEP 1201, 094 (2012), arXiv:1105.1162.

[19] B. S. Kim (2012), arXiv:1202.6062.

[20] X. Dong, S. Harrison, S. Kachru, G. Torroba, and H. Wang (2012), arXiv:1201.1905.

[21] J.-P. Wu and H.-B. Zeng, JHEP 1204, 068 (2012), arXiv:1201.2485.

[22] R.-G. Cai and A. Wang, Phys. Rev. D70, 084042 (2004), arXiv:hep-th/0406040.

[23] W.-J. Li and J.-P. Wu (2012), arXiv:1203.0674.

[24] B. Gouteraux, J. Smolic, M. Smolic, K. Skenderis, and M. Taylor, JHEP 1201, 089 (2012), arXiv:1110.2320.

[25] N. Iizuka and K. Maeda (2012), arXiv:1204.3008.

[26] E. Perlmutter, JHEP 02, 013 (2011), arXiv:1006.2124.

[27] K. Narayan (2012), arXiv:1202.5935.

[28] P. Dey and S. Roy (2012), arXiv:1204.4858.

[29] M. Cadoni, S. Mignemi, and M. Serra, Phys.Rev. D84, 084046 (2011), arXiv:1107.5979.

[30] M. Dehghani, R. Mann, and R. Pourhasan, Phys.Rev. D84, 046002 (2011), arXiv:1102.0578.

[31] S. Kachru, X. Liu, and M. Mulligan, Phys.Rev. D78, 106005 (2008), arXiv:0808.1725.

[32] G. Bertoldi, B. A. Burrington, and A. W. Peet, Phys.Rev. D80, 126004 (2009), arXiv:0907.4755.

[33] G. Bertoldi, B. A. Burrington, and A. Peet, Phys.Rev. D80, 126003 (2009), arXiv:0905.3183.

[34] I. Kanitscheider and K. Skenderis, JHEP 0904, 062 (2009), arXiv:0901.1487.

[35] H. Boonstra, K. Skenderis, and P. Townsend, JHEP 9901, 003 (1999), arXiv:hep-th/9807137.

[36] L. Huijse, S. Sachdev, and B. Swingle, Phys.Rev. B85, 035121 (2012), arXiv:1112.0573.

[37] D. S. Fisher, Phys. Rev. Lett. 56, 416 (1986).

[38] E. Shaghoulian (2011), arXiv:1112.2702.

[39] N. Ogawa, T. Takayanagi, and T. Ugajin, JHEP 1201, 125 (2012), arXiv:1111.1023.

[40] P. Townsend, Phys.Lett. B148, 55 (1984).

[41] T. Torii, K. Maeda, and M. Narita, Phys.Rev. D64, 044007 (2001).

[42] T. Hertog, Phys. Rev. D74, 084008 (2006), arXiv:gr-qc/0608075.

[43] M. Cadoni, Phys.Rev. D53, 4413 (1996), arXiv:gr-qc/9510012.

[44] C. Martinez, R. Troncoso, and J. Zanelli, Phys.Rev. D70, 084035 (2004), arXiv:hep-th/0406111.

[45] G. T. Horowitz and B. Way, Phys.Rev. D85, 046008 (2012), arXiv:1111.1243.

[46] S. Harrison, S. Kachru, and H. Wang (2012), arXiv:1202.6635. 